\title{
Actividad antimicrobiana de ceftolozano-tazobactam y ceftazidima-avibactam contra bacilos gramnegativos clínicamente relevantes aislados en México
}

\author{
Rafael Martínez-Miranda, ${ }^{\text {* }}$ Mariana Gastélum-Acosta,${ }^{1}$ Paloma Guerrero-Estrada, ${ }^{1}$ \\ Rafael I. Ayala-Figueroa ${ }^{2}$ y Luis E. Osuna-Álvarez ${ }^{3}$ \\ ${ }^{1}$ Universidad Autónoma de Baja California, Facultad de Medicina, Departamento de Microbiología Clínica; ${ }^{2}$ Universidad Autónoma de Baja California, \\ Facultad de Medicina, Departamento de Estadística; ${ }^{3}$ Hospital General de Mexicali, Servicio de Pediatría. Baja California, México
}

\section{Resumen}

Introducción: Existe poca información acerca de la efectividad de las combinaciones ceftolozano/tazobactam y ceftazidima/ avibactam en cepas clínicamente relevantes aisladas en México. Objetivo: Determinar el perfil antimicrobiano de ambos antibióticos en nuestra comunidad. Método: El presente estudio de investigación fue prospectivo, descriptivo y transversal. Se incluyeron cepas clínicamente relevantes aisladas a partir de cultivos de cepa pura durante el periodo de agosto de 2018 a enero de 2019 en Mexicali, Baja California, México. Resultados: Se analizaron 74 cepas de enterobacterias y 19 cepas de Pseudomonas aeruginosa; el porcentaje de sensibilidad de ceftazidima/avibactam fue de $100 \%$ contra enterobacterias y de 72.7 \% contra Pseudomonas aeruginosa; el porcentaje de sensibilidad de ceftolozano/tazobactam fue de $90.5 \%$ para enterobacterias y de $72.7 \%$ para Pseudomonas aeruginosa. Conclusiones: Las combinaciones ceftolozano/tazobactam y ceftazidima/avibactam ofrecen buena sensibilidad antimicrobiana in vitro, tanto contra enterobacterias productoras de betalactamasas de espectro extendido como contra Pseudomonas aeruginosa. Se requieren más datos para valorar la respuesta clínica en pacientes que reciben esas combinaciones de antibióticos.

PALABRAS CLAVE: Cefalosporinas. Inhibidores de betalactamasas. Resistencia a múltiples fármacos.

\section{Ceftolozane/tazobactam and ceftazidime/avibactam antimicrobial activity against clinically relevant gram-negative bacilli isolated in Mexico}

\begin{abstract}
Introduction: There is limited information on the effectiveness of ceftolozane/tazobactam and ceftazidime/avibactam combinations on clinically relevant strains isolated in Mexico. Objective: To determine the antimicrobial profile of both antibiotic combinations in our community. Method: The present research study was prospective, descriptive and cross-sectional. Clinically relevant strains isolated from pure-strain cultures were included during the period from August 2018 to January 2019 in Mexicali, Baja California, Mexico. Results: 74 enterobacteriaceae and 19 Pseudomonas aeruginosa strains were analyzed; the percentage of sensitivity of ceftazidime/avibactam was $100 \%$ for enterobacteriaceae and $72.7 \%$ for Pseudomonas aeruginosa; the percentage of sensitivity of ceftolozane/tazobactam for enterobacteriaceae was $90.5 \%$ and $72.7 \%$ for Pseudomonas aeruginosa. Conclusions: The ceftolozane/tazobactam and ceftazidime/avibactam combinations offer good antimicrobial sensitivity in vitro, both for ESBL-producing enterobacteriaceae and Pseudomonas aeruginosa. More data are required to assess clinical response in patients receiving these antibiotic combinations.
\end{abstract}

KEY WORDS: Cephalosporins. Beta-lactamase inhibitors. Multi-drug resistance.

\footnotetext{
Correspondencia:

Fecha de recepción: 11-06-2019

*Rafael Martínez-Miranda

Fecha de aceptación: 23-07-2020

Gac Med Mex. 2020;156:604-609

E-mail: rmartinez90@uabc.edu.mx

DOI: 10.24875/GMM.19005335

Disponible en PubMed

0016-3813/๑ 2020 Academia Nacional de Medicina de México, A.C. Publicado por Permanyer. Este es un artículo open access bajo la licencia CC BY-NC-ND (http://creativecommons.org/licenses/by-nc-nd/4.0/).
} 


\section{Introducción}

La resistencia antimicrobiana de las bacterias gramnegativas se ha convertido en una de las principales causas de morbimortalidad y en un grave problema de salud pública en el mundo. ${ }^{1}$ El aumento creciente en la prevalencia de cepas productoras de betalactamasa de espectro extendido (BLEE) y carbapenemasas en los últimos años constituye un desafío debido a la facilidad de dispersión de los mecanismos de resistencia, la dificultad para elegir un tratamiento antimicrobiano adecuado y el aumento en el tiempo de estancia intrahospitalaria. ${ }^{2,3}$

Ceftolozano/tazobactam (C/T) y ceftazidima/avibactam (CZ/A) son dos combinaciones nuevas de cefalosporinas con espectro antimicrobiano ante Pseudomonas aeruginosa, así como enterobacterias productoras de BLEE y carbapenemasas de interés clínico. En 2014, ambas combinaciones de antibióticos recibieron la aprobación de la Food and Drugs Administration de Estados Unidos y en 2017, en México por la Comisión Federal para la Protección contra Riesgos Sanitarios, para el tratamiento de infecciones urinarias e intraabdominales complicadas. ${ }^{4-6}$

Existe poca información en cuanto a la efectividad de dichos antibacterianos contra cepas aisladas en México, ${ }^{7,8}$ por tal motivo el objetivo del presente proyecto de investigación fue determinar el perfil de susceptibilidad de ambos antibióticos en nuestra comunidad.

\section{Método}

El presente estudio de investigación fue prospectivo, descriptivo y transversal. Se incluyeron 93 cepas clínicamente relevantes aisladas de cultivos de cepa pura entre agosto de 2018 y enero de 2019, con participación del laboratorio MD Microbiología Clínica, S. de R.L. de C.V. (Mexicali, Baja California, México) y el laboratorio de Microbiología Clínica de la Facultad de Medicina de la Universidad Autónoma de Baja California campus Mexicali. El origen de las muestras para su análisis microbiológico fue múltiple, incluyendo orina, secreción bronquial, sangre, secreciones de heridas y abscesos, líquido sinovial, biopsias de tejidos blandos y hueso, líquido peritoneal y líquido cefalorraquídeo.

El procesamiento de las muestras biológicas se llevó a cabo de acuerdo con el manual de procedimientos de microbiología clínica de la Asociación Americana de Microbiología. ${ }^{9}$ En todos los tipos de muestras se inoculó agar gelosa sangre de carnero a $5 \%$ y agar Maconkey; en los urocultivos se agregó agar eosina azul de metileno y agar soya tripticaseína; en los cultivos de líquido cefalorraquídeo y hemocultivos se agregó agar gelosa chocolate. Las muestras de biopsia de tejido fueron maceradas en un mortero y se inoculó un caldo-infusión de cerebro-corazón de carnero para posteriormente agregar agar gelosa sangre, agar Maconkey y agar gelosa chocolate. Los cultivos se incubaron a $37^{\circ} \mathrm{C}$ durante 24 a 72 horas en condiciones aerobias y con dióxido de carbono a $5.5 \%$ (incubadora Shellab $\AA$ modelo SCO6AD, SheloLab); no se realizó incubación en condiciones anaerobias.

Una vez desarrolladas las cepas, se identificaron utilizando el sistema automatizado Vitek $2 \circledast$ Compact de Biomérieux, que utiliza tarjetas de identificación para bacilos gramnegativos y cocos grampositivos; la tarjeta de identificación de los gramnnegativos consta de 47 pruebas bioquímicas y un pocillo de control negativo. El procedimiento para el llenado de la tarjeta es el siguiente:

- Se transfieren asépticamente $3 \mathrm{~mL}$ de solución salina estéril $(0.45-0.50 \%$ de $\mathrm{NaCl}$ con $\mathrm{pH}$ de 4.5 a 7.0 ) a un tubo de poliestireno de $12 \times 75 \mathrm{~mm}$.

- Con una asa microbiológica estéril se colecta la cantidad suficiente de colonias para preparar una suspensión homogénea equivalente a 0.5 unidades de McFarland.

- Se coloca el tubo con la suspensión y la tarjeta en una gradilla especialmente diseñada para introducirla al equipo automatizado.

Para medir la actividad antimicrobiana de CZ/A y C/T se utilizó la técnica de difusión de disco en agar Mueller-Hinton, según lo estipulan los procedimientos en el documento M100-S28 ${ }^{10}$ del Instituto de Estándares Clínicos y Laboratorio de Estados Unidos; para el antibiograma del resto de los antibióticos se utilizaron las tarjetas del sistema Vitek $2 \circledR$ Compact para bacilos gramnegativos AST-XN05 (ampicilina/sulbactam, aztreonam, cefepima, cefexima, ceftriaxona, cefuroxima, cloranfenicol, colistina, detección de BLEE, levofloxacina, meropenem, minociclina, moxifloxacina, piperacilina, tetraciclina, ticarcilina/ácido clavulánico, tigeciclina, trimetoprima) y AST-N285 (amikacina, amoxicilina/ácido clavulánico, ampicilina, cefazolina, cefoxitina, ceftazidima, ceftriaxona, ciprofloxacina, doripenem, ertapenem, detección de BLEE, gentamicina, imipenem, meropenem, nitrofurantoína, piperacilina/tazobactam, trimetoprima/sulfametoxazol). El procedimiento del antibiograma automatizado 
Tabla 1. Sensibilidad de enterobacterias y Pseudomonas aeruginosa a CZ/A y C/T

\begin{tabular}{|c|c|c|c|c|}
\hline \multirow[t]{2}{*}{ Microorganismo } & \multirow[t]{2}{*}{ Tipo de muestra } & \multirow[t]{2}{*}{ Núm. de muestras } & \multicolumn{2}{|c|}{ Sensibilidad (\%) } \\
\hline & & & CZ/A & $\mathrm{C} / \mathrm{T}$ \\
\hline \multicolumn{5}{|l|}{ Enterobacterias } \\
\hline \multirow[t]{2}{*}{ Escherichia coli } & Orina & 58 & 100 & 88 \\
\hline & Otros & 4 & 100 & 100 \\
\hline \multirow[t]{2}{*}{ Klebsiella spp. } & Orina & 4 & 100 & 100 \\
\hline & Otros & 1 & 100 & 100 \\
\hline \multirow[t]{2}{*}{ Salmonella } & Orina & 0 & NP & NP \\
\hline & Otros & 2 & 100 & 100 \\
\hline \multirow[t]{2}{*}{ Enterobacter aerogenes } & Orina & 0 & NP & NP \\
\hline & Otros & 1 & 100 & 100 \\
\hline \multirow[t]{2}{*}{ Morganella morganii } & Orina & 0 & NP & NP \\
\hline & Otros & 1 & 100 & 100 \\
\hline \multirow[t]{2}{*}{ Proteus mirabilis } & Orina & 1 & 100 & 100 \\
\hline & Otros & 1 & 100 & 100 \\
\hline \multirow[t]{2}{*}{ Serratia marcescens } & Orina & 0 & NP & NP \\
\hline & Otros & 1 & 100 & 100 \\
\hline \multirow[t]{2}{*}{ Pseudomonas } & Secreción bronquial & 11 & 72.72 & 72.72 \\
\hline & Otros & 8 & 75 & 75 \\
\hline
\end{tabular}

$\mathrm{NP}=$ no se probó, $\mathrm{CZ} / \mathrm{A}=$ ceftazidima/avibactam, $\mathrm{C} / \mathrm{T}=$ ceftolozano/tazobactam

es el siguiente: a partir de una suspensión bacteriana con una turbidez de 0.5 unidades de McFarland se transfieren $14 \mu \mathrm{L}$ a un tubo de poliestireno con $3 \mathrm{~mL}$ de solución salina, posteriormente se inserta la tarjeta correspondiente del antibiograma en la gradilla con el tubo y se introduce al equipo.

La interpretación de los resultados de la microdilución automatizada y difusión de disco se sustentó en los puntos de corte del documento M100-S28.

Para el análisis estadístico se calcularon los porcentajes de susceptibilidad y resistencia. Se utilizó la prueba de McNemar para comparar la efectividad de los antibióticos considerando como significativo un valor de $\mathrm{p}<0.05$.

\section{Resultados}

Se analizaron 74 cepas de enterobacterias y 19 cepas de Pseudomonas aeruginosa. El porcentaje de sensibilidad de CZ/A fue de $100 \%$ para enterobacterias y de $72.7 \%$ para Pseudomonas aeruginosa
(Tabla 1); respecto a C/T, el porcentaje de sensibilidad fue de $90.5 \%$ para enterobacterias y de $72.7 \%$ para $P$. aeruginosa. La muestra de origen infeccioso más frecuente fue la orina, con $68.8 \%$ (Tabla 1) del total. Se encontraron 25 enterobacterias productoras de BLEE y una productora de carbapenemasa. En las Tablas 2 y 3 se muestran los porcentajes de sensibilidad de las cepas para el resto de los antibióticos probados.

\section{Discusión}

CZ/A y $\mathrm{C} / \mathrm{T}$ son nuevos antibióticos que han sido introducidos al mercado con resultados prometedores contra bacterias resistentes a múltiples fármacos. ${ }^{11} \mathrm{La}$ problemática actual respecto a la resistencia bacteriana $^{12}$ ha llegado a ser un tema de salud pública importante. Los resultados muestran que estas nuevas combinaciones de cefalosporinas con inhibidores de betalactamasas son buenas opciones al momento de tomar una decisión terapéutica en nuestro medio, 
Tabla 2. Sensibilidad de enterobacterias a antibióticos de uso clínico

\begin{tabular}{|c|c|c|c|c|c|c|c|c|}
\hline Antibiótico & Sensibilidad & $\begin{array}{l}\text { Escherichia } \\
\text { coli (62) }\end{array}$ & $\begin{array}{l}\text { Klebsiella } \\
\text { spp. (5) }\end{array}$ & $\begin{array}{c}\text { Salmonella } \\
\text { (2) }\end{array}$ & $\begin{array}{l}\text { Enterobacter } \\
\text { aerogenes (1) }\end{array}$ & $\begin{array}{c}\text { Morganella } \\
\text { morganii (1) }\end{array}$ & $\begin{array}{c}\text { Proteus } \\
\text { mirabilis (2) }\end{array}$ & $\begin{array}{c}\text { Serratia } \\
\text { marcescens (2) }\end{array}$ \\
\hline \multirow[t]{2}{*}{ AMP } & Núm. cepas & 49 & 4 & NP & NP & NP & 2 & NP \\
\hline & Sensibilidad & $24 \%$ & $0 \%$ & NP & NP & NP & $50 \%$ & NP \\
\hline \multirow[t]{2}{*}{ AMC } & Núm. muestras & 27 & 4 & NP & NP & NP & NP & NP \\
\hline & Sensibilidad & $44 \%$ & $50 \%$ & NP & NP & NP & NP & NP \\
\hline \multirow[t]{2}{*}{ SAM } & Núm. muestras & 34 & 1 & 2 & 1 & 1 & 2 & NP \\
\hline & Sensibilidad & $38.23 \%$ & $0 \%$ & $100 \%$ & $100 \%$ & $100 \%$ & $50 \%$ & NP \\
\hline \multirow[t]{2}{*}{ CXM } & Núm. muestras & 34 & 2 & 2 & 1 & NP & 2 & NP \\
\hline & Sensibilidad & $57.14 \%$ & $0 \%$ & $0 \%$ & $0 \%$ & NP & $50 \%$ & NP \\
\hline \multirow[t]{2}{*}{ CXM axetil } & Núm. muestras & 32 & 1 & 2 & 1 & NP & 2 & NP \\
\hline & Sensibilidad & $56 \%$ & $0 \%$ & $0 \%$ & $0 \%$ & NP & $50 \%$ & NP \\
\hline \multirow[t]{2}{*}{$\mathrm{CRO}$} & Núm. muestras & 62 & 5 & 2 & 1 & 1 & 1 & NP \\
\hline & Sensibilidad & $64.51 \%$ & $40 \%$ & $100 \%$ & $100 \%$ & $100 \%$ & $100 \%$ & NP \\
\hline \multirow[t]{2}{*}{ FEP } & Núm. muestras & 35 & 3 & 2 & 1 & 1 & 2 & NP \\
\hline & Sensibilidad & $68.57 \%$ & $33 \%$ & $100 \%$ & $100 \%$ & $100 \%$ & $50 \%$ & NP \\
\hline \multirow[t]{2}{*}{ ETP } & Núm. muestras & 51 & 5 & NP & NP & 1 & 2 & NP \\
\hline & Sensibilidad & $98 \%$ & $100 \%$ & NP & NP & $100 \%$ & $100 \%$ & NP \\
\hline \multirow[t]{2}{*}{ MEM } & Núm. muestras & 62 & 5 & 2 & 1 & 1 & 2 & NP \\
\hline & Sensibilidad & $96.77 \%$ & $100 \%$ & $100 \%$ & $100 \%$ & $100 \%$ & $100 \%$ & NP \\
\hline \multirow[t]{2}{*}{ LVX } & Núm. muestras & 62 & 5 & 2 & 1 & 1 & 2 & NP \\
\hline & Sensibilidad & $56.45 \%$ & $80 \%$ & $100 \%$ & $100 \%$ & $0 \%$ & $50 \%$ & NP \\
\hline \multirow[t]{2}{*}{ CIP } & Núm. muestras & 58 & 4 & 2 & 1 & 1 & 2 & NP \\
\hline & Sensibilidad & $56.89 \%$ & $100 \%$ & $100 \%$ & $100 \%$ & $0 \%$ & $50 \%$ & NP \\
\hline \multirow[t]{2}{*}{ AMK } & Núm. muestras & 55 & 3 & NP & NA & 1 & 2 & NP \\
\hline & Sensibilidad & $98.18 \%$ & $100 \%$ & NP & NA & $100 \%$ & $100 \%$ & NP \\
\hline \multirow[t]{2}{*}{ GEN } & Núm. muestras & 49 & 4 & NP & NA & 1 & 2 & NP \\
\hline & Sensibilidad & $72.00 \%$ & $75 \%$ & NP & NA & $0 \%$ & $50 \%$ & NP \\
\hline \multirow[t]{2}{*}{ SXT } & Núm. muestras & 51 & 4 & 2 & 1 & 1 & 2 & NP \\
\hline & Sensibilidad & $65 \%$ & $25 \%$ & $100 \%$ & $100 \%$ & $0 \%$ & $100 \%$ & NP \\
\hline \multirow[t]{2}{*}{ NIT } & Núm. muestras & 53 & 4 & NP & NP & NA & 2 & NP \\
\hline & Sensibilidad & $84.90 \%$ & $25 \%$ & NP & NP & NA & $50 \%$ & NP \\
\hline \multirow[t]{2}{*}{ FOS } & Núm. muestras & 54 & 4 & NP & NP & NA & 2 & NP \\
\hline & Sensibilidad & $94.44 \%$ & $25 \%$ & NP & NP & NA & $100 \%$ & NP \\
\hline
\end{tabular}

$\mathrm{NP}=$ no se probó, $\mathrm{AMP}=$ ampicilina, $\mathrm{AMC}=$ amoxicilina/ácido clavulánico, $\mathrm{SAM}=$ ampicilina/sulbactam, $\mathrm{CXM}=$ cefuroxima, $\mathrm{CRO}=$ ceftriaxona, $\mathrm{FEP}=$ cefepima, $\mathrm{ETP}=$ ertapenem $\mathrm{MEM}=$ meropenem, $\mathrm{LVX}=$ levofloxacino, $\mathrm{CIP}=$ ciprofloxacino, $\mathrm{AMK}=$ amikacina, $\mathrm{GEN}=$ gentamicina, $\mathrm{SXT}=$ trimetoprima/sulfametoxazol, $\mathrm{NIT}=$ nitrofurantoína, $\mathrm{FOS}=$ fosfomicina. 
Tabla 3 . Sensibilidad de Pseudomonas aeruginosa a antibióticos antipseudomonas

\begin{tabular}{|c|c|c|}
\hline Antibióticos & Sensibilidad & Pseudomonas $(n=19)$ \\
\hline \multirow[t]{2}{*}{ Ticarcilina/AC } & Núm. cepas & 16 \\
\hline & Sensibilidad & 12.5 \\
\hline \multirow[t]{2}{*}{ Piperacilina/T } & Núm. muestras & 19 \\
\hline & Sensibilidad & 52.63 \\
\hline \multirow[t]{2}{*}{ Ceftazidima } & Núm. muestras & 17 \\
\hline & Sensibilidad & 64.7 \\
\hline \multirow[t]{2}{*}{ Cefepima } & Núm. muestras & 18 \\
\hline & Sensibilidad & 61.11 \\
\hline \multirow[t]{2}{*}{ Imipenem } & Núm. muestras & 18 \\
\hline & Sensibilidad & 38.88 \\
\hline \multirow[t]{2}{*}{ Meropenem } & Núm. muestras & 19 \\
\hline & Sensibilidad & 42.1 \\
\hline \multirow[t]{2}{*}{ Levofloxacina } & Núm. muestras & 19 \\
\hline & Sensibilidad & 36.84 \\
\hline \multirow[t]{2}{*}{ Ciprofloxacina } & Núm. muestras & 19 \\
\hline & Sensibilidad & 31.57 \\
\hline \multirow[t]{2}{*}{ Amikacina } & Núm. muestras & 17 \\
\hline & Sensibilidad & 58.82 \\
\hline \multirow[t]{2}{*}{ Colistina } & Núm. muestras & 18 \\
\hline & Sensibilidad & 94.44 \\
\hline
\end{tabular}

por esta razón decidimos enviar la información obtenida hasta ahora como comunicación breve.

CZ/A mostró sensibilidad superior en enterobacterias productoras de BLEE en comparación con $\mathrm{C} / \mathrm{T}$ (100\% versus $80 \%$ ). Ambas combinaciones de fármacos mostraron actividad antimicrobiana superior en comparación con los antibióticos de uso común en enterobacterias, como las quinolonas $(100 \%$ versus $56 \%$ ), cefalosporinas (100\% versus $63.4 \%$ ) y penicilinas (100\% versus $24 \%$ ). En las cepas aisladas de Pseudomonas, CZ/A y C/T presentaron mayor actividad (sensibilidad de $73.6 \%$ ) en comparación con antibióticos carbapenémicos (meropenem $42.1 \%$, imipenem $38.8 \%$ ) y antipseudomonas como amikacina (58.82\%), piperacilina/tazobactam (52.3\%), ceftazidima $(64.7 \%)$ y cefepima $(61.1 \%)$, con lo que se sitúan como los antibióticos más sensibles, solo después de colistina (94.4\%).

Además de la comparación que se realizó con los nuevos fármacos de interés, este estudio muestra al mismo tiempo la elevada resistencia de las cepas a varios antibióticos considerados de primera elección en bacterias gramnegativas tales como la cefuroxima, ceftriaxona, levofloxacino y ciprofloxacino, con aproximadamente $40 \%$ de resistencia de Escherichia coli y $50 \%$ de Pseudomonas aeruginosa a carbapenemas, $40 \%$ a penicilinas antipseudomonas y alrededor de $30 \%$ de resistencia a aminoglucósidos. Estos patrones de resistencia observados en nuestra comunidad son similares a los reportados en publicaciones recientes ${ }^{12}$ y deben ser considerados por el clínico al momento de tomar una decisión terapéutica.

En conclusión, la combinaciones CZ/A y C/T tienen una buena respuesta in vitro, sobre todo de enterobacterias productoras de BLEE y Pseudomonas aeruginosa. CZ/A y C/T al ser comparados con los antibióticos convencionalmente utilizados en la práctica clínica para infecciones causadas por enterobacterias, no mostraron una diferencia estadísticamente significativa respecto a meropenem $(p=1$ versus $p=0.625$, respectivamente), pero sí fueron claramente superiores a ceftriaxona $(p<0.01$ versus $p<0.01$ ). En cuanto a Pseudomonas aeruginosa, debido al reducido número de cepas no es pertinente aseverar la superioridad estadística de CZ/A y C/T en comparación con otros antibióticos antipseudomonas como colistina y meropenem.

Si bien CZ/A y C/T pueden ser muy útiles en el manejo de microorganismos gramnegativos altamente resistentes, actualmente los datos disponibles para respaldar su eficacia clínica en México aún son limitados. Otro aspecto por considerar es el costo económico, el cual puede ser casi del doble si se compara con el de otros antibióticos que mostraron actividad antimicrobiana similar, como son los carbapenémicos y aminoglucósidos.

Exhortamos al uso juicioso de los datos de susceptibilidad obtenidos con esta investigación, ya que la frecuencia de aislamiento de cepas patógenas, así como la resistencia bacteriana pueden variar considerablemente según las diferentes regiones geográficas, incluso entre hospitales de la misma ciudad. Es elemental conocer la situación epidemiológica específica del lugar donde nos ubicamos, las principales bacterias aisladas en la comunidad, así como 
sus patrones de resistencia, lo cual permitirá orientar el tratamiento antibiótico con menor margen de error. ${ }^{13}$

Una limitación de la presente investigación fue no disponer de los reactivos para realizar microdilución de $\mathrm{CZ/A}$ y $\mathrm{C} / \mathrm{T}$, por que no fue posible comparar la potencia in vitro de ambos antibióticos contra las cepas identificadas.

\section{Agradecimientos}

Agradecemos el valioso apoyo brindado por la química farmacobióloga Martha Dorado del Río en el procesamiento de las muestras de origen clínico.

\section{Conflicto de intereses}

Los autores declaran no tener conflicto de intereses alguno.

\section{Financiamiento}

Los autores no recibieron patrocinio para llevar a cabo este artículo.

\section{Responsabilidades éticas}

Protección de personas y animales. Los autores declaran que para esta investigación no realizaron experimentos en seres humanos ni en animales.

Confidencialidad de los datos. Los autores declaran que en este artículo no aparecen datos de pacientes.
Derecho a la privacidad y consentimiento informado. Los autores declaran que en este artículo no aparecen datos de pacientes.

\section{Bibliografía}

1. Yezli S, Shibl AM, Memish ZA. The molecular basis of $\beta$-lactamase production in gram-negative bacteria from Saudi Arabia. J Med Microbiol. 2015;64:127-136.

2. Aly M, Balkhy $\mathrm{HH}$. The prevalence of antimicrobial resistance in clinical isolates from Gulf Corporation Council countries. Antimicrob Resist Infect Control. 2012;1:26.

3. Aquino-Andrade A, Mérida-Vieyra J, Arias-de la Garza E, Arzate-Barbosa P, de Colsa-Ranero A. Carbapenemase-producing Enterobacteriaceae in Mexico: report of seven non-clonal cases in a pediatric hospital. BMC Microbiol. 2018;18:38.

4. D Leuthner K, Kullar R, Jayakumar B, Hewlett DA, Nguyen T, Puzniak L. Real-world evaluation of ceftolozane/tazobactam (C/T) use and clinical outcomes at an academic medical center in Las Vegas. Open Forum Infect Dis. 2017:4:2.

5. Liscio JL, Mahoney MV, Hirsch EB. Ceftolozane/tazobactam and ceftazidime/avibactam: two novel $\beta$-lactam/ $\beta$-lactamase inhibitor combination agents for the treatment of resistant Gram-negative bacterial infections. Int J Antimicrob Agents. 2015:46:266-271.

6. Merck [sitio web]. Estados Unidos: Highlights of prescribing information. Zerbaxa ${ }^{\circledR}$ (ceftolozane and tazobactam) for injection, for intravenous use; 2014.

7. Carrazco I. Susceptibilidad a ceftolozane/tazobactam en cepas de pseudomonas aeruginosa resistentes a carbapenémicos en CMN La Raza. Enferm Infecc Microbiol Clin. 2018;38:280.

8. Hernández AM, Avilés RM, Castellanos CM. Susceptibilidad in vitro de Pseudomonas aeruginosa. Multidrogorresiste te a ceftolozano/tazobactam en muestras clínicas de pacientes pediátricos. Enferm Infecc Microbiol Clin. 2018:38:92.

9. American Society for Microbiology. Manual of Clinical Microbiology. Décima primera edición. EE. UU: American Society for Microbiology Press; 2015.

10. Clinical and Laboratory Standards Institute (CLSI). M100: performance standards for antimicrobial susceptibility testing. Vigésima octava edición. EE. UU.: Clinical and Laboratory Standards Institute; 2018.

11. Alatoom A, Elsayed $H$, Lawlor $K$, AbdelWareth L, El-Lababidi $R$ Cardona $L$, et al. Comparison of antimicrobial activity between ceftolozane-tazobactam and ceftazidime-avibactam against multidrug-resistant isolates of Escherichia coli, Klebsiella pneumoniae, and Pseudomonas aeruginosa. Int J Infect Dis. 2017;62:39-43.

12. Garza-González E, Morfín-Otero R, Mendoza-Olazarán S, Bocanegra-lbarias P, Flores-Treviño S, Rodríguez-Noriega E, et al. A snapshot of antimicrobial resistance in Mexico. Results from 47 centers from 20 states during a six-month period. PLoS One. 2018;14:e0209865.

13. Snydman DR, McDermott LA, Jacobusb LV. Activity of ceftolozane-tazobactam against a broad spectrum of recent clinical anaerobic isolates. Antimicrob Agents Chemother. 2014;58:1218-1223. 